\title{
PRISMA: A robust and intuitive tool for high-throughput processing of chemical spectra
}

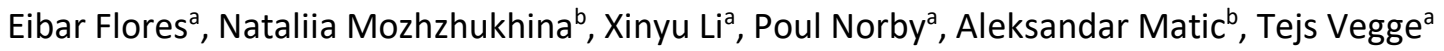 \\ a: Department of Energy Conversion and Storage, Technical University of Denmark, DK-2800 Kgs. Lyngby, \\ Denmark. \\ b: Department of Physics, Chalmers University of Technology, SE 412 96, Göteborg, Sweden.
}

\begin{abstract}
The popularization of high-throughput spectroscopies to characterize functional materials requires the simultaneous development of new analysis tools to efficiently process large numbers of measurements into scientifically meaningful observables. Here we introduce PRISMA, an open-source tool to rapidly analyze hundreds of spectra in a semi-automated way. PRISMA follows a human-in-the-loop workflow, where the user interacts with an intuitive graphical user interface (GUI) to control multiple steps in the spectrum analysis process: trimming, baseline correction, and peak fitting. The user tunes the analysis in real-time and applies the optimal parameters to all spectra, outputting processed results in an easy-toread csv format within seconds. Crucially, the tuned parameters are stored to guarantee the full reproducibility of the analysis. We describe the functionalities implemented in PRISMA and test its capabilities with three experimental cases relevant to the study of electrochemical energy storage and conversion devices: temperature-dependent Raman measurement of phase transitions, a linear Raman mapping of a graphite composite electrode, and an operando X-ray diffraction experiment of $\mathrm{LiNiO}_{2} \mathrm{Li}$-ion electrode. Even if X-ray diffraction is not a spectroscopic technique, diffraction patterns are represented as one-dimensional arrays of counts equally suitable for analysis with PRISMA. The case studies demonstrate the robustness of the app and its ability to unearth insightful trends in peak parameters, which are easier to represent, interpret and further analyze with more advanced techniques.
\end{abstract}

\section{Introduction}

The rapid development of advanced tools for characterizing electrochemical energy storage and conversion devices (EESCDs) are popularizing high-throughput (HT) experiments. In HT experiments, a large number of measurements are acquired from different locations on a spatially-heterogeneous system (i.e., mapping) or from a system perturbed by temperature, electric potential, time, pressure, etc., to monitor its state, evolution, and degradation as a function of the perturbation variable. In particular, in situ and operando characterizations are increasingly used because the complex nature of the electrochemical environments requires studying functional materials within the working environment and while they operate. Typically, these experiments involve building a functional device (e.g., an electrolyzer, a Li-ion battery, etc.) into a special cell that enables operating the device and characterizing its components through special windows transparent to the probing radiation.

A significant portion of the portfolio of techniques applied to study EESCDs[1-5] output a one-dimensional array of (photon/electron) counts whose indexes correspond to a scanning variable, e.g., energies in absorption spectroscopies, wavenumbers in vibrational spectroscopies, diffraction angles in diffractionbased techniques. We use the term spectrum to refer to the aforementioned array, acknowledging that while diffraction-based techniques are fundamentally different from spectroscopies, they share the same output data format. 
$\mathrm{HT}$ experiments can generate hundreds or even thousands of spectra as a function of a perturbation variable (e.g. time, temperature, etc.), which are often modulated by instrumental, measurement, and physical factors that introduce undesirable data features, e.g., high noise, outliers, and unstable baselines. Conventionally, every spectrum is manually processed with basic techniques using commercial software (e.g., smoothing for noise reduction and outlier removal, polynomial baseline fitting to anchored points in the spectra), to then be represented into cascade or contour plots for drawing conclusions. When the experiment outputs several spectra, individual peak fitting is also useful to identify trends in peak parameters and deconvolute signals. However, as the number of spectra per experiment grows, a manual approach becomes not only cumbersome but also susceptible to arbitrary user decisions that can bias or negatively affect the reproducibility of the analysis. The automation of the whole analysis pipeline becomes necessary, but it is often difficult to implement in commercial software. Hence, many research groups resort to developing in-house and custom-made code that is rarely published, and even when it is, the code appears inaccessible to researchers with no programming experience. Ideally, any such tool should fulfill two main goals. The first is to assemble a library of spectrum-processing functions into an open codebase that can be operated, updated and populated by a broad community. The second goal is to make these tools accessible to researchers with no programming experience, via an intuitive graphical user interface (GUI). While there are notable tools available for processing spectra, [6-9] these either lack GUIs, or they implement components built on proprietary dependencies, or do not support highthroughput analysis, or the code is compiled hence restricted from community development.

Here, we present PRISMA, an app based on Python for reproducible, high-throughput processing of multiple spectra in an automatable way. A prototypic version of the code was already demonstrated to be critical in the elucidation of dynamic phenomena on Li-ion battery electrodes[10-12] and interfaces.[13] We now build from previous versions to produce an externalizable app addressing the scientific need for open, reliable, user-friendly and fast analysis tools to extract meaningful patterns from (time, voltage, temperature, spatially)-dependent spectroscopic experiments. In the following sections we provide an overview of the code implementation initially supporting three main processing functionalities, designed as modular building blocks to facilitate future implementations of novel analysis techniques. In addition, we describe the general analysis workflow and present three case studies testing the robustness of the PRISMA app and demonstrating its role in analyzing phase transitions, chemical maps and structural changes in temperature-, spatially- and time-dependent experiments, respectively. Finally, we discuss the value of PRISMA as a research tool and directions for future development.

\section{Code Implementation and functionality}

PRISMA is designed with the vision of the BIG-MAP project, which aims to accelerate the discovery of new battery materials.[14] The development of interoperable, community-wide research tools is essential within this vision.[15] Accordingly, the design of the app is guided by the goals of facilitating the implementation, deployment and interoperability of spectrum-processing tools. Python and its numerical computing library Numpy are highly popular for scientific programming,[16] and so they are obvious choices for implementing spectrum processing tools. In addition, we provide a GUI built with the ipywidgets module and rendered with the voilà package. For developers, the widget package enables building apps capable of running on the web browser using Jupyter Lab.[17-19] At the same time, endusers (e.g. researchers) interact directly with clickable components in the app and so they are not burdened with implementation details. Two key design choices are made to support code interoperability. First, spectra and its processing tools are decorated with metadata identifiers such that they can be 
integrated to, e.g., ontology[20] and provenance management frameworks.[21] Second, the GUI components are independent from all core functionalities. In this way, the spectrum processing functions (e.g., the baseline subtraction algorithm) can be operated not only by the complementary GUI here developed, but also as an individual package and by any external code via an Application Programming Interface (API), opening the possibility of integrating the code with third-party apps and web services. Last, the code is published open source code to facilitate collaborative, third-party development of new functionalities. A complete documentation of the codebase can be found in reference [22].

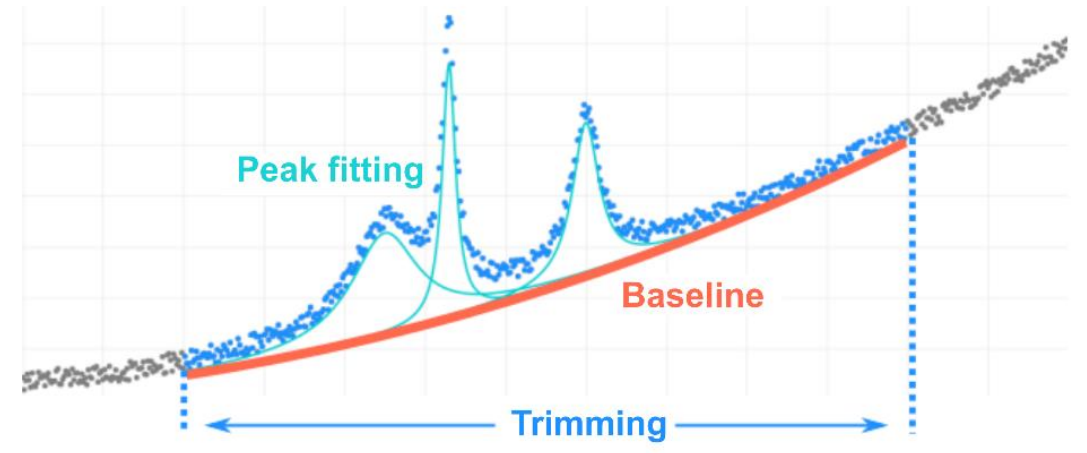

Figure 1. Representation of the three main functionalities currently implemented in PRISMA on a synthetic spectrum.

PRISMA currently supports three main processing functionalities, summarized in Fig. 1.

Spectrum trimming: Cut a spectrum at the intervals defined by the user.

Baseline correction: Fits a baseline curve to a spectrum based on the Asymmetric Least Squares smoothing method (AsLS) developed by Eilers and Boelens. $[23,24]$ While there are multiple approaches for baseline correction of spectra[25], which could also readily be implemented as part of PRISMA's codebase, we prefer the AsLS method due to its relative simplicity, fast computation performance and excellent baseline estimation.[24] Furthermore, unlike traditional approaches, the user does not need to manually indicate anchor points in the spectrum to estimate its baseline, thus minimizing user intervention and facilitating the automation of the correction process. The method finds a curve being i) smooth, ii) faithful to the spectrum, and iii) asymmetrically penalizing positive residuals, where the analytical peaks are found. The optimal baseline signal $z_{i}$ is found by minimizing iteratively the penalized least squares function:

$$
S=\sum_{i} w_{i}\left(y_{i}-z_{i}\right)^{2}+\lambda \sum_{i}\left(\Delta^{2} z_{i}\right)^{2}
$$

where $y_{i}$ represents the spectrum counts and $\Delta^{2} z_{i}=z_{i}-2 z_{i-1}+z_{i-2}$ quantifies the smoothness of the baseline. The user tunes the baseline via two main parameters:

- Penalty $p$ : accentuate the relative importance (the weights $w_{i}$ ) of data points at the base of the spectrum. Larger $p$ include higher-valued data points in the fitting (Fig. 2a).

- Smoothness $\lambda$ : control the smoothness of the baseline curve. Larger $\lambda$ result in flatter curves (Fig. $2 b)$. 

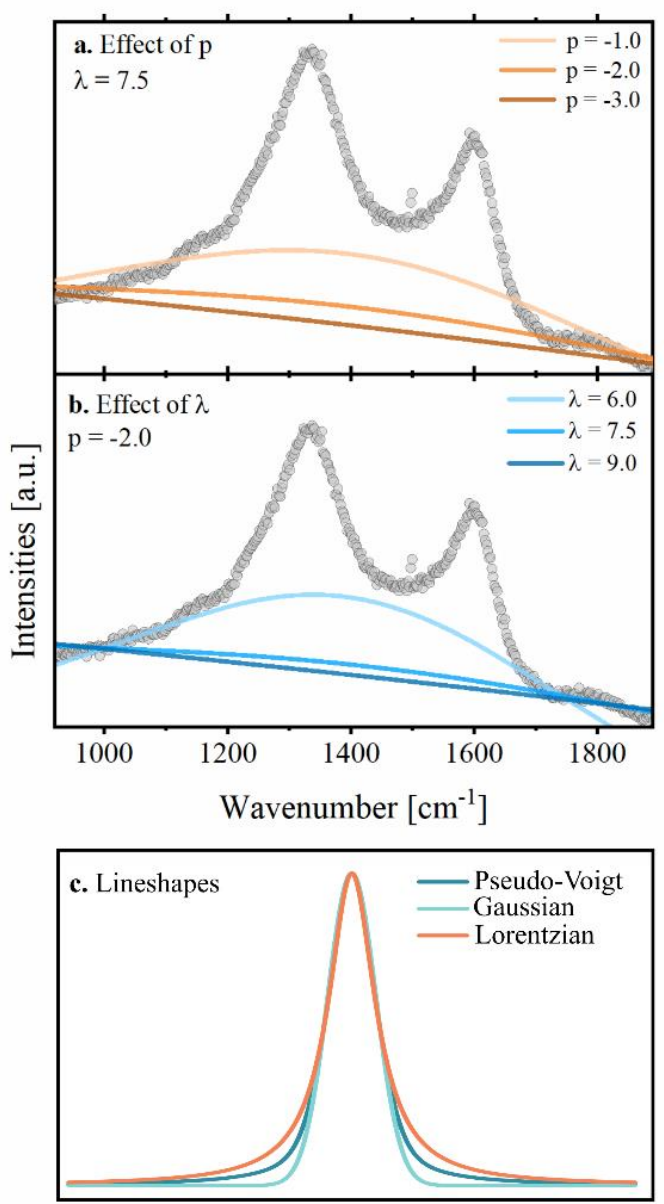

Figure 2. Effect of a. penalty ( $p)$ and $b$. smoothness $(\lambda)$ parameters on the baseline of the Raman spectrum of hard carbon. c. Peak profiles implemented in PRISMA.

Peak fitting: Models a spectrum as a combination of peak profiles defined by an analytical expression. For each peak, the user specifies an upper and a lower bound defining the peak neighborhood, along with a maximum bound for the peak's width. The code then fits the complete spectrum as a sum of peak profiles using the Trust Region Reflective Algorithm as implemented in the SciPy package.[26] The resulting fitting parameters (e.g., position, height and half width at half maximum) are used to characterize the spectrum. The peak profiles included in PRISMA (Table 1) describe the most commonly found peak profiles, which are naturally (Lorentzian), partially (Pseudo-Voigt) or heavily (Gaussian) broadened by the measurement conditions. $[27,28]$ Figure $2 c$ highlights differences among peak profiles: Lorentzian profiles exhibit longer tails compared to Gaussians, while Pseudo-Voigt profiles with $50 \%$ Gaussian/Lorentzian mix result in an intermediate profile. 
Table 1: Three peak profiles implemented in PRISMA, defined by the peak height $h$, the half-width at half-maximum $w$ and the center $p$.

\begin{tabular}{|l|c|c|c|}
\hline Peak profile & Description & Analytical expression & Peak profile Area \\
\hline Lorentzian & $\begin{array}{c}\text { Spectral bands where natural } \\
\text { broadening is } \\
\text { dominant.[27,29] }\end{array}$ & $L(x)=\frac{h w^{2}}{(x-p)^{2}+w^{2}}$ & $A=\pi h w$ \\
\hline Gaussian & $\begin{array}{c}\text { Spectral bands dominated by } \\
\text { measurement broadening. }\end{array}$ & $G(x)=h e^{\frac{-\ln (2)(x-p)^{2}}{w^{2}}}$ & $A=\sqrt{\frac{\pi}{\ln (2)}} h w$ \\
\hline $\begin{array}{l}\text { Pseudo-Voigt } \\
\text { (50\% Lorentzian) }\end{array}$ & $\begin{array}{c}\text { Intermediate case between } \\
\text { Lorentzian and Gaussian } \\
\text { profiles. }\end{array}$ & $P_{50 \%}(x)=\frac{1}{2} L(x)+\frac{1}{2} G(x)$ & $A=\frac{1}{2} \pi h w\left(1+\sqrt{\frac{1}{\pi \ln (2)}}\right)$ \\
\hline
\end{tabular}

\section{Methodology: Case Studies}

Case 1: Melting transition of ethylene carbonate. Temperature-dependent Raman measurements were carried out on $1 \mathrm{ml}$ of ethylene carbonate (E-lyte) in a glass vial placed inside a custom-built temperature control chamber. The temperature ramp was set to heat from $25^{\circ} \mathrm{C}$ to $65^{\circ} \mathrm{C}$ within 7 hours, using a digital temperature controller Eurotherm. The Raman spectra were acquired with Bruker MultiRAM FT-Raman spectrometer fitted with a liquid nitrogen cooled Ge-diode detector, probing with a $1064 \mathrm{~nm}$ laser wavelength at $400 \mathrm{~mW}$ of laser power. A total of 150 spectra were recorded over 100 scans at $4 \mathrm{~cm}^{-1}$ resolution, each at $0.26^{\circ} \mathrm{C}$ temperature intervals.

Case 2: Raman mapping of a composite graphite electrode. The composite graphite electrode was manufactured by CIDITEC using the following composition: 15-4 Graphite (IMERYS), 94 wt\%; C45 Carbon Black (IMERYS), 2wt\%; BM451B SBR (ZEON), 2 wt\%; Carboxymethyl cellulose binder: Walocel CRT2000(DOW), 2 wt\%. The Raman spectra were acquired with a HORIBA LabRam HR Evolution confocal Raman spectrometer using 50x long working distance objective and $488 \mathrm{~nm}$ laser wavelength (filtered to $5 \%$ laser power, c.a. $2.5 \mathrm{~mW}$ ), $300 \mathrm{~nm}$ grating and 150 seconds total acquisition time. The confocal hole was set to $200 \mathrm{~nm}$. The line scan was recorded over $60 \mu \mathrm{m}$ with a spectrum recorded every $0.5 \mu \mathrm{m}$.

Case 3. Operando X-ray diffraction of $\mathrm{LiNiO}_{2}$. The $\mathrm{LiNiO}_{2}$ slurry was prepared by mixing $\mathrm{LiNiO}_{2}$ active material powder (BASF), PVDF (Sigma Aldrich) and carbon black (IMERYS, $\geq 96 \%$ ) with a weight ratio of 93: 3.5: 3.5 in N-Methyl-2-pyrrolidone solvent. The slurry was coated on an aluminium foil and dried at $120^{\circ} \mathrm{C}$ overnight. The dried material was scratched from the foil and cold-pressed into a pellet $(7 \mathrm{~mm}$ in diameter and $152 \mu \mathrm{m}$ in thickness) under 0.75 tons pressure and subsequently dried at $120^{\circ} \mathrm{C}$ in a vacuum oven inside a glovebox overnight. A modified electrochemical-cell (ECC-OPTO) was assembled inside the glovebox using the pellet as cathode, a lithium foil with a diameter of $9 \mathrm{~mm}$ as anode and LP57 (E-lyte, $1 \mathrm{M} \mathrm{LiPF}_{6}$ in 3:7 EC/EMC) as electrolyte for operando X-ray diffraction (XRD) measurements; further details about the cell and its assembly can be found in reference [30]. Diffraction data were recorded on a Rigaku Smartlab diffractometer in reflection geometry with a CuKa $\left(\lambda_{1}=1.54059, \lambda_{2}=1.54441 \AA ; \lambda_{2} / \lambda_{1}\right.$ intensity ratio $=0.5$ ) radiation source. The diffraction data were collected every 16.5 minutes within a $2 \theta$ range of $10-130^{\circ}$ with a step size of $0.01^{\circ}$.

\section{App use}


The typical workflow when using PRISMA to analyze a sequence of dependent spectra is illustrated in the code documentation [22] and the Supplementary video. In short, the user:

1. Chooses a pipeline, i.e., a pre-defined recipe of spectrum processing steps.

2. Uploads the file(s) with the spectra. PRISMA offers three parsers able to load spectra in specific formats; i.e., as individual .txt files, as a single .txt file and as a single .csv file. For more details on the accepted file formats see Supplementary Table T1.

3. Selects a spectrum to visualize.

4. Tunes the processing parameters until reaching a visually satisfactory fit. For baseline correction, the parameters are the trimming interval and the $\lambda$ and $p$ parameters of the fit; for peak fitting these are the number of peak profiles, their bounding neighborhood and a maximum width limit.

5. Inspects whether the parameters enable satisfactory fits of other spectra or if they need further tuning.

6. Iterates between steps 4 and 5 selecting multiple spectra and evaluating the results by visual inspection, until finding satisfactory parameters that apply to most spectra.

7. Runs a high-throughput processing of all spectra with the chosen parameters.

8. Downloads the results for further analysis and plotting.

PRISMA also supports downloading individual .csv files of a processed spectrum that include baseline and peak profiles. This functionality renders the app suitable to analyze ex-situ, post-mortem and, in general, spectra generated from unrelated experiments. In this case, the previous workflow includes a 4.1 step to download the results of an individual spectrum and stops at step 4 after all spectra have been processed and downloaded individually.

In the following sections we demonstrate three representative battery examples using PRISMA. The cases studies aim to test the user experience, robustness to different datasets and illustrate the scientific value of PRISMA in accelerating the processing of raw spectra data.

Case 1. Melting transition of ethylene carbonate. Ethylene carbonate (EC) is an organic solvent widely used as component of Li-ion battery electrolytes due to its crucial role in forming stable solid electrolyte interphases (SEI).[31] EC is solid at room temperature but melts at $36.3^{\circ} \mathrm{C}$. [32] We have recorded the Raman spectra of EC as a function of temperature, from $25^{\circ} \mathrm{C}$ to $65^{\circ} \mathrm{C}$ in order to monitor the melting transition. Figure $3 a$ shows a contour plot of the resulting temperature-dependent spectra within the interval 2825 to $3150 \mathrm{~cm}^{-1}$ where $\mathrm{CH}_{2}$ vibrations appear[33] (complete range in Supplementary information Fig. S1). Figure $3 \mathrm{~b}$ show samples of solid and liquid EC for comparison. Evidently, the spectra of solid and liquid phases remain stable except within the transition region between 35 and $40{ }^{\circ} \mathrm{C}$, were most bands shift and change intensity. 
a. $\mathrm{CH}_{2}$ region

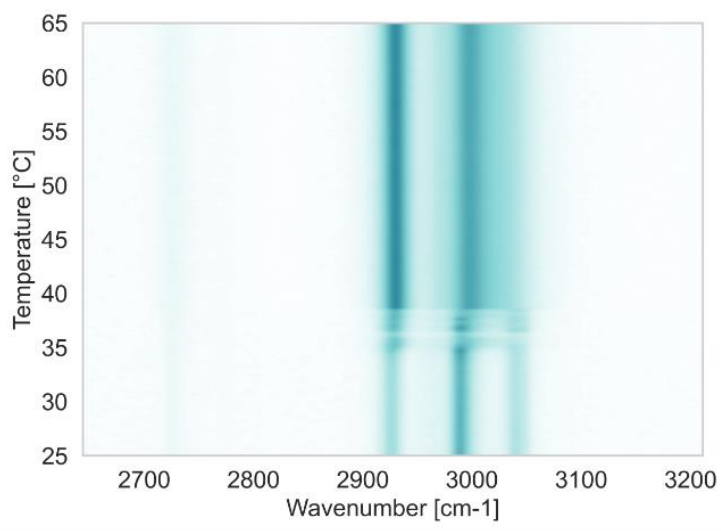

b. Peak fits

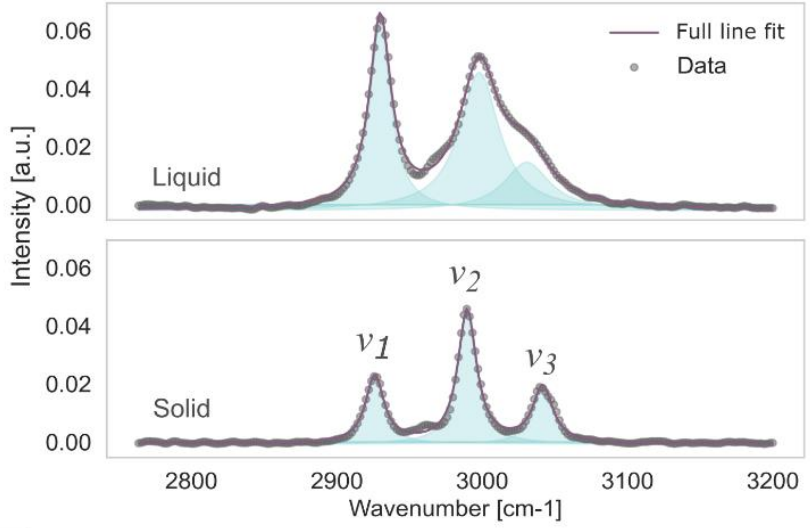

Figure 3. a. Contour plot depicting Raman spectra of ethylene carbonate as a function of temperature in the range 2825 to $3150 \mathrm{~cm}^{-1}$. b. Raman spectrum and the PRISMA app fitted curve of liquid and solid ethylene carbonate.

The spectra within the 2825 to $3150 \mathrm{~cm}^{-1}$ wavenumber region was processed with PRISMA for baseline correction and to obtain peak fitting parameters based on three Lorentzian spectral contributions, named for convenience $v_{1}, v_{2}$ and $v_{3}$. Figure 4 shows the peak position and intensity trends as a function of temperature. As the transition temperature is approached, the bands shift in a non-monotonic fashion: $v_{3}$ from 3040 to $3030 \mathrm{~cm}^{-1}, v_{2}$ from 2990 to $2998 \mathrm{~cm}^{-1}$ and $v_{1}$ from 2926 to $2930 \mathrm{~cm}^{-1}$. At the same time, the peak intensities of the two higher wavenumber $\mathrm{C}$-H stretch bands vary very slightly, while the intensity of the lower wavenumber $\mathrm{C}-\mathrm{H}$ stretching mode increases almost 3-fold during the state transition. The spectroscopic changes likely originate from the structural rearrangement and concomitant symmetry changes the EC molecule undergoes upon melting. [33-36] Notably, all trends display a clear discontinuity at the transition temperature.

a. Peak positions

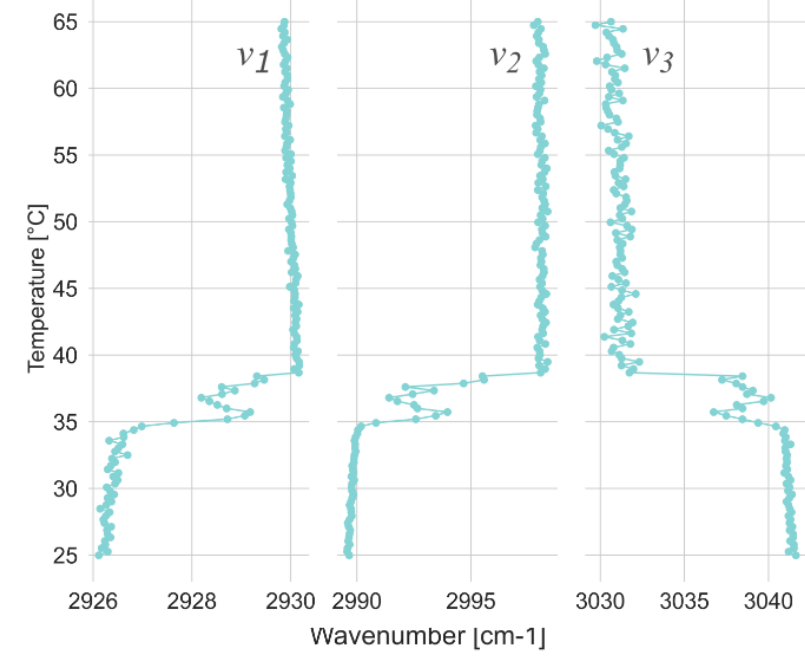

b. Peak intensities

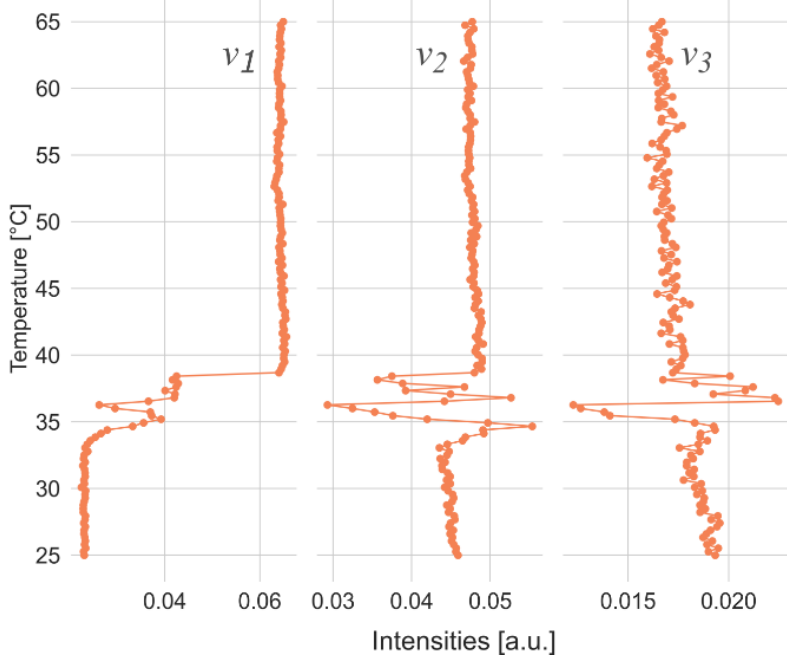

Figure 4. Plots of the parametres for the PRISMA fitted spectral components a. positions and $b$. intensities of the $C-H$ stretch region of ethylene carbonate, as a function of temperature. 
PRISMA enables processing of the 100+ spectra generated in the experiment within a few minutes. The analysis results in feature-rich peak parameters trends, which constitute an alternative and more detailed representation of the system's behavior. For instance, the intensity discontinuities in our case study would be difficult to observe in conventional spectrum representations such as contour (e.g., Figure 3a) or waterfall plots, but they are clearly visible in the representation of Figure 4a-b. In a broader sense, the peak parameter representation enabled by tools like PRISMA highlights peak shifts, intensity changes and trend discontinuities; all of which can prove crucial in understanding the solid-liquid, liquid-gas, crystalline-amorphous transitions, etc., inherent to the operation and stability of a wide range of functional materials.

Case 2. Raman mapping of a composite graphite electrode. Graphite is the anode material of choice for the Li-ion battery[37]. Typically, the electrode is fabricated as a composite of micrometre-sized graphite particles mixed with a conductive additive and coated with a binder onto a copper foil current collector (see image on the figure $5 \mathrm{~d}$ ). Here, we performed line scan of the graphite electrode along $60 \mu \mathrm{m}$, recording a Raman spectrum every $0.5 \mu \mathrm{m}$, which oversamples the scanning line given that the lateral resolution is $2.4 \mu \mathrm{m}$. As the laser probes the electrode along the line shown in the figure $5 \mathrm{~d}$, it encounters graphite particles fully or partially covered with the carbon conductive additive and binder, and particle interspaces, i.e. voids to be filled with electrolyte. The subtle differences between the Raman signatures of graphite and the carbon additive introduce spectroscopic changes along the scanning direction.

Graphite, as well as other carbonaceous materials, is typically characterized by D and G Raman-active bands that appear within the $1000-1700 \mathrm{~cm}^{-1}$ region and correspond to disordered and ordered domains in the graphitic structure, respectively.[38] The ratio between the two bands depends on multiple variables, including incident laser wavelength, degree of disorder, defects, surface groups, and crystalline size.[39] Disordered graphitic materials also exhibit a D' band located at a slightly higher wavenumber than the $G$ band and typically appear as a shoulder of the latter. In some cases, three bands are not sufficient for an adequate fitting of spectra from a composite graphite electrode.[40] A five-peak fitting model with $D_{1}(D), D_{2}\left(D^{\prime}\right), D_{3}, D_{4}$ and $G$ bands has been suggested to provide more accurate fits and insight into the structure and chemical nature of carbonaceous materials.[41,42] The additional two bands, $D_{3}$ and $D_{4}$ have been assigned to presence of amorphous carbon from the conductive additive, surface groups and impurities; $[41,42]$ Figures $5 e$ and $5 f$ illustrate the 5-peak fitting model using Lorentzian peak profiles, from which the intensities are extracted and followed across the electrode. 


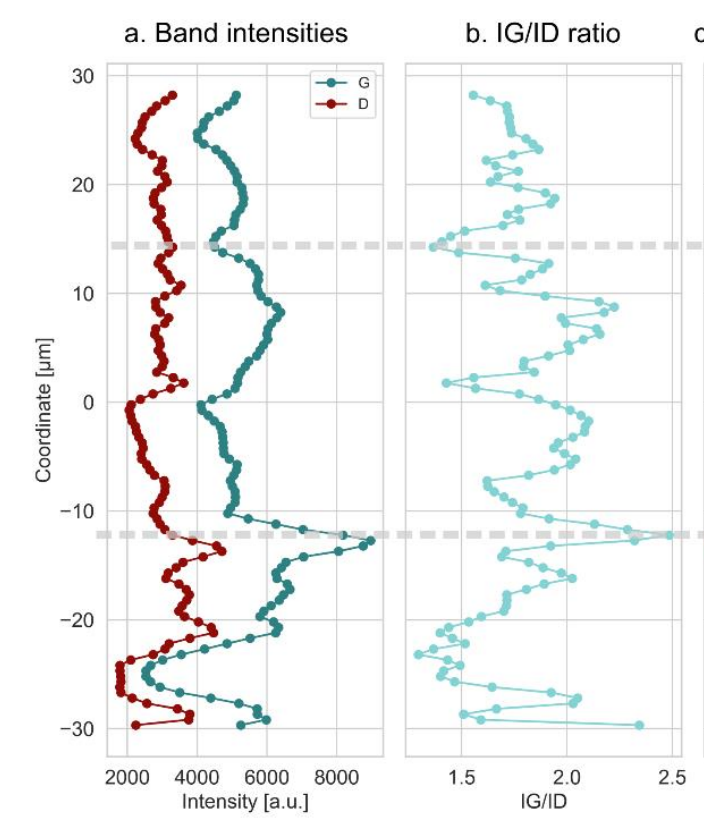

c. Point-wise covariance

d. Optical image of electrode
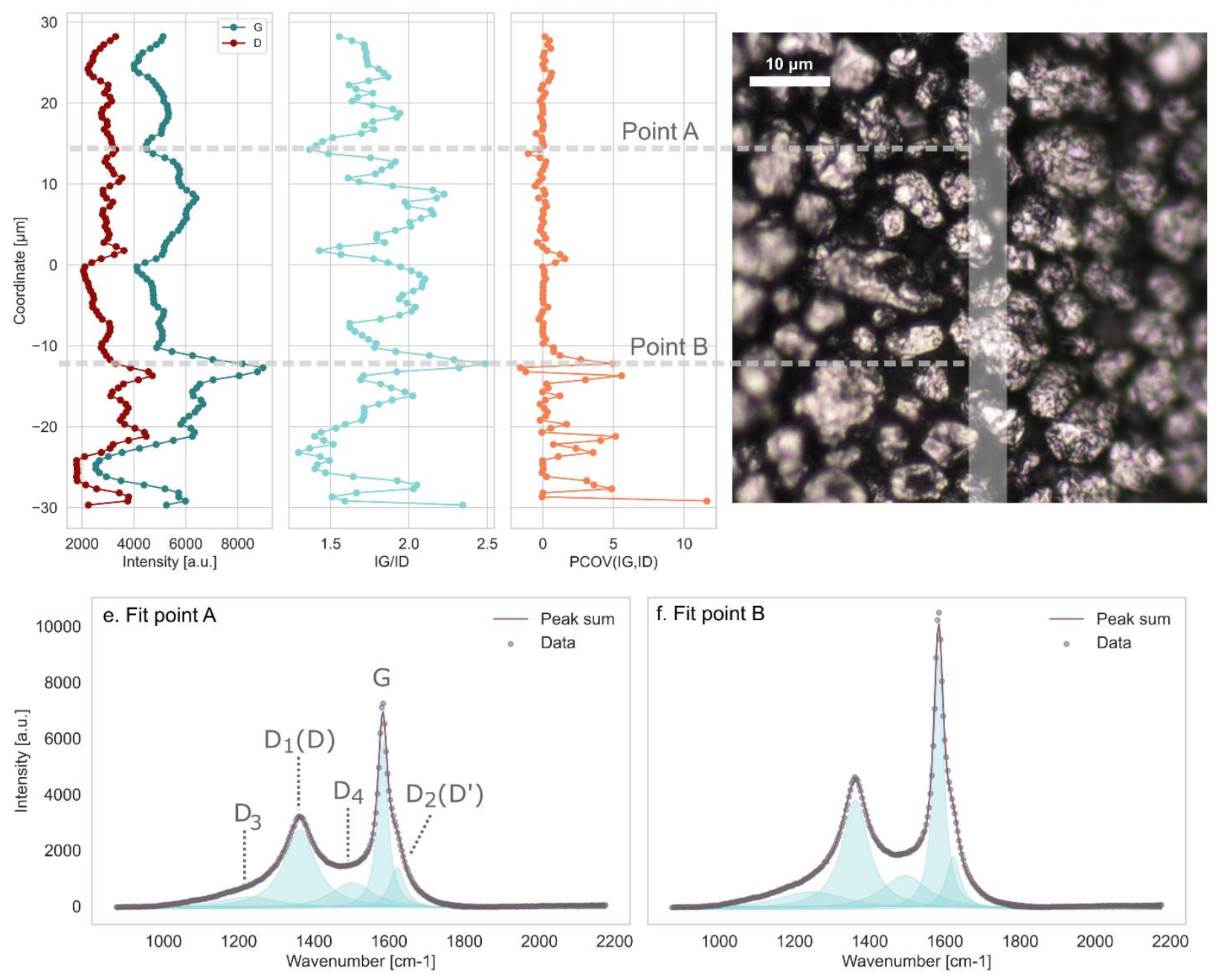

Figure 5. Peak fitting of the line scan case study. $a$. Fitted $D$ and $G$ band intensities, $b$. Intensity ratio trend, and $c$. point-wise covariance between $I_{G}$ and $I_{D}$ numerical differences, as a function of the linear coordinate. $d$. Aligned optical image of the electrode, highlighting the line scan. Baseline-subtracted spectra fitted with the 5-Lorentzian band model from $e$. Point $A$ and $f$. Point $B$ along the linear mapping.

Figure $5 a$ shows the fitted $D$ and $G$ intensities along the line scan, aligned with the optical image in Figure $5 \mathrm{~d}$. Given there is no autofocus functionality enabled during the scan, the recorded intensities are subjected to both i) topographic contrasts, originating from elevation changes relative to the axial position of the laser probe and ii) chemical contrasts, resulting from the mixture between graphitic and disordered carbon. We expect topographic changes (voids and hills) to affect the intensities of both $D$ and $G$ bands equally. Instead, chemical changes (order and disordered carbon) would predominantly affect the $I_{G}$ to $I_{D}$ intensity ratio.[43] Based on these assumptions, we attempt deconvoluting topographic from chemical contrasts by computing two derived metrics: i) the $I_{G} / I_{D}$ intensity ratio, and ii) a point-wise covariance between the numerical differences of $I_{G}$ and $I_{D}$ trends. The covariance metric was chosen because its property to increase when $I_{G}$ and $I_{D}$ change simultaneously and decrease when $I_{G}$ and $I_{D}$ changes are uncorrelated. In other words, high $I_{G} / I_{D}$ ratios indicate graphite-rich regions, while high covariances are a proxy for elevation changes along the scanning direction. 
The $I_{G} / I_{D}$ trend in Figure $5 b$ aids identifying highly crystalline graphitic regions; for instance, Point $A$ coincides with a particle interspace that is filled with the disordered conductive carbon, according to the low $I_{G} / I_{D}$ ratio. In contrast, the $I_{G} / I_{D}$ ratio is high at Point $B$, where a big graphitic particle is clearly identified from the optical image. Interestingly, the covariance trends in Figure $5 \mathrm{C}$ appear decoupled from the $I_{G} / I_{D}$ ratios, suggesting a successful deconvolution of chemical and topological contrasts. The covariance trends indicate a relatively flat region between 30 and $-10 \mu \mathrm{m}$ suggesting that particle interspaces are filled with conductive additive. Conversely, elevation changes appear between -10 and $-30 \mu \mathrm{m}$ and thus suggest the presence of large particles partially covered with conductive additive.

From the scientific perspective, this case study further illustrates the value of analyzing peak trends instead of raw spectra. First, the baseline substraction step removes the underlying intensity contributions from the baseline and enables exclusive monitoring of the Raman-active signals. Second, derivative metrics computed from the peak trends might offer insightful descriptions, e.g., topographic contrasts, chemical heterogeneities, etc. From the user's perspective, the challenging spectroscopic analysis of carbonaceous materials illustrates another advantage of PRISMA: its GUI enables a fluent feedback between tuning the analysis and visualizing the results. The user is able to quickly explore many analysis constraints -baseline parameters, number and constrains of peaks- in the pursuit for a fit both visually accurate and scientifically sound.

Case 3. Operando X-ray diffraction of $\mathrm{LiNiO}_{2}$. Challenges on the world supply of cobalt are fueling research efforts on the search for stable, high-energy and Co-free cathode materials for Li-ion batteries.[44,45] $\mathrm{LiNiO}_{2}$ (LNO) is being revisited as a prominent candidate since it shares the layered crystal structure of already commercialized $\mathrm{LiCoO}_{2}$ and $\mathrm{NMC}\left(\mathrm{LiNi}_{x} \mathrm{Mn}_{y} \mathrm{Co}_{2} \mathrm{O}_{2}\right)$ materials and deliver higher energy density; however, a series of cycling-dependent phase transitions are believed to limit its cycling reversibility. [46] Therefore, it is important to characterize the phase diagram and the structural relation between the observed phases in order to identify the structural culprits of cell degradation. We have performed operando XRD experiments during delithiation and lithiation of a LNO/Li half-cell. The evolution of the XRD patterns from the $\mathrm{Li}_{x} \mathrm{NiO}_{2}$ electrode during galvanostatic cycling are characterized by multiple transitions among four phases (cf. Figure 6a): the initial hexagonal structure $(\mathrm{H} 1)$ transforms into a monoclinic phase (M) during delithiation. By further delithiation another hexagonal phase $(\mathrm{H} 2)$ is formed, which at high degree of delithiation transforms into an hexagonal phase $(\mathrm{H} 3)$ with a significantly smaller interlayer distance. The reactions were shown to be reversible upon subsequent lithiation. $[47,48]$ 

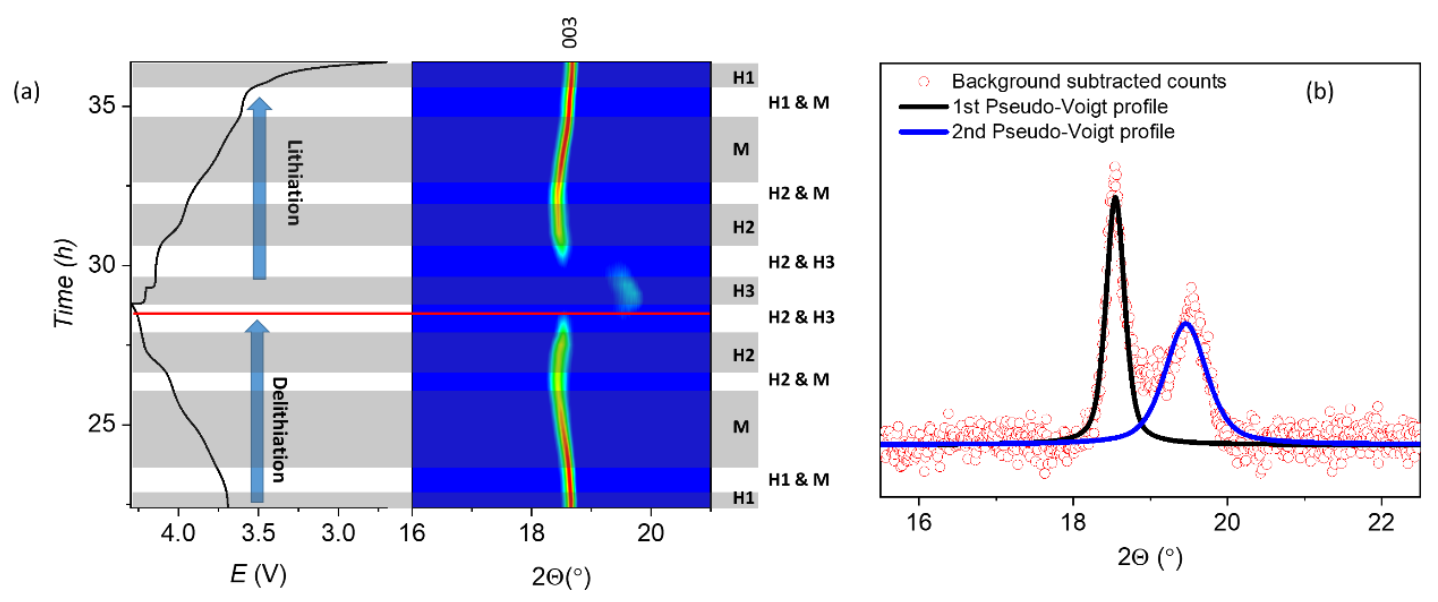

Figure 6. (a) XRD contour plot of the 003 reflection of $\mathrm{Li}_{x} \mathrm{NiO}_{2}$ and voltage profile as a function of time during delithiation and lithiation. Single-phase regions are indicated using grey rectangle shade and biphasic regions are indicated between these single-phase regions; (b) example of PRISMA-fitted XRD pattern within H2 and H3 biphasic region (Red line indicates the selected XRD pattern)

The phase transitions of $\mathrm{Li}_{x} \mathrm{NiO}_{2}$ upon delithiation are accompanied with changes of lattice parameters and relative fraction of distinct phases, which will reflect on peak positions and intensities, respectively. These changes originate from a combined effect of the lithium-content $(x)$ and $\mathrm{Ni}^{3+}$ to $\mathrm{Ni}^{4+}$ oxidation during $\mathrm{Li}_{x} \mathrm{NiO}_{2}$ delithiation. $[49,50]$ Particularly, the $c$ lattice parameter is known to undergo a dramatic decrease when $\mathrm{H} 2$ phase transforms to $\mathrm{H} 3$ because the Li-containing intralayer collapses at low lithium-content $(x)$.[51] The large difference in lattice parameter $c$ between $\mathrm{H} 2$ and $\mathrm{H} 3$ phases manifests as a shift of the 003 reflection of $\mathrm{Li}_{x} \mathrm{NiO}_{2}$ in XRD patterns.

We performed a HT fitting of the 003 reflection using PRISMA to obtain background-subtracted XRD patterns and peak fitting parameters including peak positions ( $d$-spacings) and heights. The baseline subtraction procedure enabled decoupling the effects of interfering signals from the glassy carbon used as cell window (see Supp. Fig. S2). Two Pseudo-Voigt peak profiles were adapted to fit the 003 reflection: the first fits the 003 reflection at low angle $\left(18.4^{\circ} \leq 2 \theta \leq 18.7^{\circ}\right)$, corresponding to $\mathrm{H} 1 / \mathrm{M} / \mathrm{H} 2$ phase regions; and the second fits the $\mathrm{H} 3$ phase at high angle $\left(19.5^{\circ} \leq 2 \theta \leq 19.7^{\circ}\right)$. Figure $6 \mathrm{~b}$ shows an example of PRISMA-fitted XRD pattern within the $\mathrm{H} 2 / \mathrm{H} 3$ biphasic region. Evolutions of $d$-spacings and peak heights for the 003 reflection as a function of time upon delithiation and lithiation are presented in Figure $7 a$ and $b$, respectively. The obtained evolution of $d$-spacings is highly consistent with that from Rietveld refinements and clearly shows a significant $\mathrm{d}_{003}$ change between $\mathrm{H} 1$ and $\mathrm{H} 3$ phases. In addition, the peak intensity trends show the $\mathrm{H} 3$ phase appearing at cell voltages larger than $4.1 \mathrm{~V} \mathrm{vs.} \mathrm{Li}^{+} / \mathrm{Li}$ and becoming the dominant phase thereafter. The presence of a contracted H3 phase has been associated with intra-and inter-particle mechanical stresses leading eventually to particle cracking and the rapid degradation of the active material.[48,52-54] 


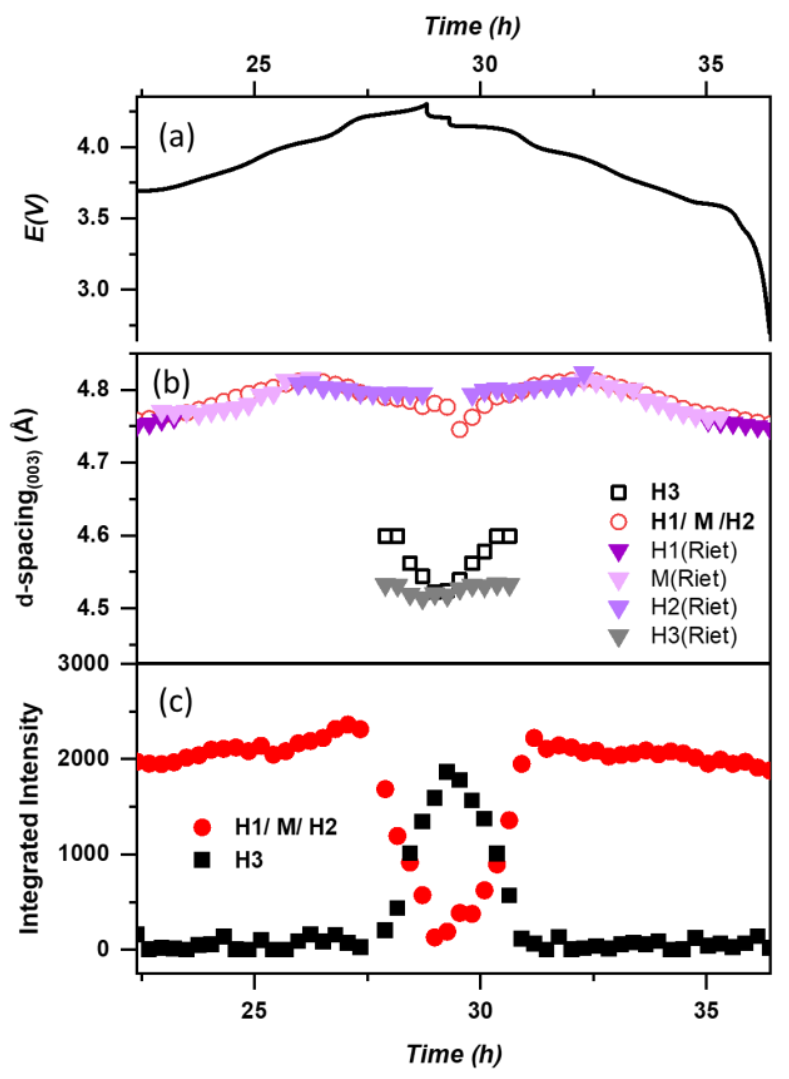

Figure 7. (a) Voltage profile as a function of time during delithiation and lithiation; (b) Comparison of obtained dspacing(003) evolution upon delithiation and lithiation from Rietveld refinement and PRISMA fitting; (c) PRISMAfitted peak heights of the (003) reflection upon delithiation and lithiation.

PRISMA is by no means a replacement for structural refinement for phase identification. However, when analyzing hundreds of diffraction patterns, individual refinement becomes cumbersome and time consuming. Sequential refinement might automate the analysis of large number of patterns, but choosing the right initial structural model can be challenging for multi-phasic systems such as LNO. Here PRISMA becomes a complementary tool for quickly analyzing many patterns within a narrow region of interest, e.g. the 003 reflection for LNO reflecting cycling-dependent changes on the $d_{003}$ spacing. Moreover, using PRISMA as an element in automated structural analysis of complex powder diffraction data from in situ and operando experiments could enable autonomous experimental control by providing on-line and realtime interpretation of crystallographic properties.

\section{Conclusions and outlook}

We introduce PRISMA as a general purpose and user-friendly tool for high-throughput analysis of spectra. The app abstracts away implementation details and enables researchers to focus on applying their knowledge and intuition for analyzing hundreds of spectra. Users execute an intuitive workflow essentially loading the raw files, tuning analysis parameters, visually inspecting the results and running a highthroughput processing step - all executed over few minutes. The graphical user interface is intuitive and provides a flexible control over the analysis parameters, which are saved for guaranteeing the reproducibility of the results. The app accepts general spectroscopic files (including diffraction patterns) with widely-used text formats, and outputs the results as .csv files that can be easily loaded to most 
scientific plotting software. We test the current version of PRISMA with three experimental cases frequently encountered in electrochemical energy storage research. The cases demonstrate i) the robustness of PRISMA to analyze spectroscopic and diffraction data with varying degrees of noise and baselines and ii) the benefits of analyzing peak trends for understanding the physicochemical phenomena underpinning the operation of functional materials.

More broadly, PRISMA also aims to become an interoperable platform connecting the development of spectra-processing tools, to their implementation, prototyping, and final deployment for general users. In this sense, we envision adding new tools i) to complement the current analysis pipelines (e.g. with outlier rejection and smoothing), or ii) to automate processing of spectra with fully autonomous algorithms, $[7,25,55,56]$ or iii) to implement data-driven analysis via dimensionality reduction techniques[57,58] and trained machine learning models.[59]

\section{CRediT authorship contribution statement}

Eibar Flores: Conceptualization, Software development, Writing - original draft, Formal Analysis, Investigation, Visualization, Project Administration. Nataliia Mozhzhukhina: Resources, Experiments, Investigation, Writing - Review \& editing for cases 1 and 2. Xinyu Li: Resources, Experiments, Investigation, Writing - Review \& editing for case 3. Poul Norby: Supervision, Writing - Review \& editing for case 3. Aleksandar Matic: Supervision, Writing - Review \& editing for cases 1 and 2. Tejs Vegge: Funding acquisition, Conceptualization, Writing - Review \& editing.

\section{Declaration of competing interest}

The authors declare that they have no known competing financial interests or personal relationships that could have appeared to influence the work reported in this paper.

\section{Acknowledgements}

This project has received funding from the European Union's Horizon 2020 research and innovation initiative under grants agreement No 957189 (BIG-MAP) and No 957213 (BATTERY 2030+).

\section{Supplementary Data}

Supplementary information with Table of supported file-formats, contour Raman plots of ethylene carbonate and contour Raman plots of original and baseline-corrected XRD pattern of $\mathrm{LiNiO}_{2}$. Supplementary Video tutorial for using the app.

\section{References}

[1] J. Lu, T. Wu, K. Amine, State-of-the-art characterization techniques for advanced lithium-ion batteries, Nat. Energy. 2 (2017). https://doi.org/10.1038/nenergy.2017.11.

[2] Z. Lin, P.L. Taberna, P. Simon, Advanced analytical techniques to characterize materials for electrochemical capacitors, Curr. Opin. Electrochem. 9 (2018) 18-25.

https://doi.org/10.1016/j.coelec.2018.03.004.

[3] A. Arvay, E. Yli-Rantala, C.H. Liu, X.H. Peng, P. Koski, L. Cindrella, P. Kauranen, P.M. Wilde, A.M. Kannan, Characterization techniques for gas diffusion layers for proton exchange membrane fuel cells - A review, J. Power Sources. 213 (2012) 317-337.

https://doi.org/10.1016/j.jpowsour.2012.04.026. 
[4] X. Li, H.-Y. Wang, H. Yang, W. Cai, S. Liu, B. Liu, In Situ/Operando Characterization Techniques to Probe the Electrochemical Reactions for Energy Conversion, Small Methods. 2 (2018) 1700395. https://doi.org/10.1002/smtd.201700395.

[5] P.P.R.M.L. Harks, F.M. Mulder, P.H.L. Notten, In situ methods for Li-ion battery research: A review of recent developments, J. Power Sources. 288 (2015) 92-105. https://doi.org/10.1016/j.jpowsour.2015.04.084.

[6] Lucas Hermann Negri, PeakUtils, (2017). https://doi.org/10.5281/zenodo.887917.

[7] T.M. James, M. Schlösser, R.J. Lewis, S. Fischer, B. Bornschein, H.H. Telle, Automated quantitative spectroscopic analysis combining background subtraction, cosmic ray removal, and peak fitting, Appl. Spectrosc. 67 (2013) 949-959. https://doi.org/10.1366/12-06766.

[8] P. Candeloro, E. Grande, R. Raimondo, D. Di Mascolo, F. Gentile, M.L. Coluccio, G. Perozziello, N. Malara, M. Francardi, E. Di Fabrizio, Raman database of amino acids solutions: A critical study of Extended Multiplicative Signal Correction, Analyst. 138 (2013) 7331-7340. https://doi.org/10.1039/c3an01665j.

[9] F. Menges, Spectragryph-optical spectroscopy software, (2017). https://effemm2.de/spectragryph/index.html.

[10] E. Flores, N. Vonrüti, P. Novák, U. Aschauer, E.J. Berg, Elucidation of $\mathrm{Li}_{x} \mathrm{Ni}_{0.8} \mathrm{Co}_{0.15} \mathrm{Al}_{0.05} \mathrm{O}_{2}$ Redox Chemistry by Operando Raman Spectroscopy, Chem. Mater. 30 (2018) 4694-4703. https://doi.org/10.1021/acs.chemmater.8b01384.

[11] E. Flores, P. Novák, U. Aschauer, E.J. Berg, Cation Ordering and Redox Chemistry of Layered NiRich Li xNi1-2 yCo yMn yO2: An Operando Raman Spectroscopy Study, Chem. Mater. 32 (2020) 186-194. https://doi.org/10.1021/acs.chemmater.9b03202.

[12] E. Flores, N. Mozhzhukhina, U. Aschauer, E.J. Berg, Operando Monitoring the Insulator-Metal Transition of LiCoO 2 , ACS Appl. Mater. Interfaces. 13 (2021) 22540-22548. https://doi.org/10.1021/acsami.1c04383.

[13] N. Mozhzhukhina, E. Flores, R. Lundström, V. Nyström, P.G. Kitz, K. Edström, E.J. Berg, Direct Operando Observation of Double Layer Charging and Early Solid Electrolyte Interphase Formation in Li-Ion Battery Electrolytes, J. Phys. Chem. Lett. 11 (2020) 4119-4123.

https://doi.org/10.1021/acs.jpclett.0c01089.

[14] T. Vegge, J.-M. Tarascon, K. Edström, Toward Better and Smarter Batteries by Combining Al with Multisensory and Self-Healing Approaches, Adv. Energy Mater. 11 (2021) 2100362. https://doi.org/10.1002/AENM.202100362.

[15] I.E. Castelli, D.J. Arismendi-Arrieta, A. Bhowmik, I. Cekic-Laskovic, S. Clark, R. Dominko, E. Flores, J. Flowers, K. Ulvskov Frederiksen, J. Friis, A. Grimaud, K. Vels Hansen, L.J. Hardwick, K. Hermansson, L. Königer, H. Lauritzen, F. Le Cras, H. Li, S. Lyonnard, H. Lorrmann, N. Marzari, L. Niedzicki, G. Pizzi, F. Rahmanian, H. Stein, M. Uhrin, W. Wenzel, M. Winter, C. Wölke, T. Vegge, Data Management Plans: the Importance of Data Management in the BIG-MAP Project, Batter. Supercaps. (2021) batt.202100117. https://doi.org/10.1002/batt.202100117.

[16] C.R. Harris, K.J. Millman, S.J. van der Walt, R. Gommers, P. Virtanen, D. Cournapeau, E. Wieser, J. Taylor, S. Berg, N.J. Smith, R. Kern, M. Picus, S. Hoyer, M.H. van Kerkwijk, M. Brett, A. Haldane, J.F. del Río, M. Wiebe, P. Peterson, P. Gérard-Marchant, K. Sheppard, T. Reddy, W. Weckesser, H. 
Abbasi, C. Gohlke, T.E. Oliphant, Array programming with NumPy, Nature. 585 (2020) 357-362. https://doi.org/10.1038/s41586-020-2649-2.

[17] ipywidgets, (2021). https://github.com/jupyter-widgets/ipywidgets.

[18] T. Kluyver, B. Ragan-Kelley, F. Pérez, B. Granger, M. Bussonnier, J. Frederic, K. Kelley, J. Hamrick, J. Grout, S. Corlay, P. Ivanov, D. Avila, S. Abdalla, C. Willing, Jupyter Notebooks - a publishing format for reproducible computational workflows, Position. Power Acad. Publ. Play. Agents Agendas - Proc. 20th Int. Conf. Electron. Publ. ELPUB 2016. (2016) 87-90. https://doi.org/10.3233/978-1-61499-649-1-87.

[19] J. Tuloup, E. Al, Voila, (2021). https://github.com/voila-dashboards/voila.

[20] H. Happel, S. Seedorf, Applications of ontologies in software engineering, in: Work. Semat. Web Enabled Softw. Eng. ISWC, 2006: pp. 5-9.

http://citeseerx.ist.psu.edu/viewdoc/download?doi=10.1.1.89.5733\&rep=rep1\&type=pdf.

[21] S.P. Huber, S. Zoupanos, M. Uhrin, L. Talirz, L. Kahle, R. Häuselmann, D. Gresch, T. Müller, A. V. Yakutovich, C.W. Andersen, F.F. Ramirez, C.S. Adorf, F. Gargiulo, S. Kumbhar, E. Passaro, C. Johnston, A. Merkys, A. Cepellotti, N. Mounet, N. Marzari, B. Kozinsky, G. Pizzi, AiiDA 1.0, a scalable computational infrastructure for automated reproducible workflows and data provenance, Sci. Data. 7 (2020) 300. https://doi.org/10.1038/s41597-020-00638-4.

[22] E. Flores, PRISMA, (2021). https://github.com/BIG-MAP/PRISMA.

[23] P.H.C. Eilers, A perfect smoother, Anal. Chem. 75 (2003) 3631-3636. https://doi.org/10.1021/ac034173t.

[24] P.H.C. Eilers, H.F.M. Boelens, Baseline Correction with Asymmetric Least Squares Smoothing, 2005. http://www.science.uva.nl/ hboelens/publications/draftpub/Eilers_2005.pdf.

[25] G. Schulze, A. Jirasek, M.M.L. Yu, A. Lim, R.F.B. Turner, M.W. Blades, Investigation of Selected Baseline Removal Techniques as Candidates for Automated Implementation, Appl. Spectrosc. 59 (2005) 545-574. https://doi.org/10.1366/0003702053945985.

[26] P. Virtanen, R. Gommers, T.E. Oliphant, M. Haberland, T. Reddy, D. Cournapeau, E. Burovski, P. Peterson, W. Weckesser, J. Bright, S.J. van der Walt, M. Brett, J. Wilson, K.J. Millman, N. Mayorov, A.R.J. Nelson, E. Jones, R. Kern, E. Larson, C.J. Carey, I. Polat, Y. Feng, E.W. Moore, J. VanderPlas, D. Laxalde, J. Perktold, R. Cimrman, I. Henriksen, E.A. Quintero, C.R. Harris, A.M. Archibald, A.H. Ribeiro, F. Pedregosa, P. van Mulbregt, SciPy 1.0: fundamental algorithms for scientific computing in Python, Nat. Methods. 17 (2020) 261-272. https://doi.org/10.1038/s41592-0190686-2.

[27] W. Demtröder, Widths and Profiles of Spectral Lines, in: Laser Spectrosc., Springer, Berlin, 2003: pp. 59-95. https://doi.org/10.1007/978-3-662-05155-9_3.

[28] T. Váczi, A new, simple approximation for the deconvolution of instrumental broadening in spectroscopic band profiles, Appl. Spectrosc. 68 (2014) 1274-1278. https://doi.org/10.1366/1307275.

[29] R.J. Meier, On art and science in curve-fitting vibrational spectra, Vib. Spectrosc. 39 (2005) 266269. https://doi.org/10.1016/j.vibspec.2005.03.003. 
[30] D.D. Blanchard, M. Slagter, In operando Raman and optical study of lithium polysulphides dissolution in Lithium-Sulfur Cells with carrageenan binder, J. Phys. Energy. 18 (2021) 119-123. https://doi.org/10.1088/2515-7655/ac0b37.

[31] K. Xu, Nonaqueous Liquid Electrolytes for Lithium-Based Rechargeable Batteries, Chem. Rev. 104 (2004) 4303-4418. https://doi.org/10.1021/cr030203g.

[32] M.S. Ding, K. Xu, T.R. Jow, Liquid-Solid Phase Diagrams of Binary Carbonates for Lithium Batteries, J. Electrochem. Soc. 147 (2000) 1688. https://doi.org/10.1149/1.1393419.

[33] M. Masia, M. Probst, R. Rey, Ethylene Carbonate-Li + : A Theoretical Study of Structural and Vibrational Properties in Gas and Liquid Phases, J. Phys. Chem. B. 108 (2004) 2016-2027. https://doi.org/10.1021/jp036673w.

[34] B. Fortunato, P. Mirone, G. Fini, Infrared and Raman spectra and vibrational assignment of ethylene carbonate, Spectrochim. Acta Part A Mol. Spectrosc. 27 (1971) 1917-1927. https://doi.org/https://doi.org/10.1016/0584-8539(71)80245-3.

[35] J.L. Allen, O. Borodin, D.M. Seo, W.A. Henderson, Combined quantum chemical/Raman spectroscopic analyses of Li+ cation solvation: Cyclic carbonate solvents-Ethylene carbonate and propylene carbonate, J. Power Sources. 267 (2014) 821-830.

https://doi.org/10.1016/j.jpowsour.2014.05.107.

[36] B. Klassen, R. Aroca, M. Nazri, G.A. Nazri, Raman Spectra and Transport Properties of Lithium Perchlorate in Ethylene Carbonate Based Binary Solvent Systems for Lithium Batteries, J. Phys. Chem. B. 102 (1998) 4795-4801. https://doi.org/10.1021/jp973099d.

[37] J. Asenbauer, T. Eisenmann, M. Kuenzel, A. Kazzazi, Z. Chen, D. Bresser, The success story of graphite as a lithium-ion anode material - fundamentals, remaining challenges, and recent developments including silicon (oxide) composites, Sustain. Energy Fuels. 4 (2020) 5387-5416. https://doi.org/10.1039/DOSE00175A.

[38] M.A. Pimenta, G. Dresselhaus, M.S. Dresselhaus, L.G. Cançado, A. Jorio, R. Saito, Studying disorder in graphite-based systems by Raman spectroscopy, Phys. Chem. Chem. Phys. 9 (2007) 1276-1290. https://doi.org/10.1039/B613962K.

[39] A.C. Ferrari, J. Robertson, Interpretation of Raman spectra of disordered and amorphous carbon, Phys. Rev. B. 61 (2000) 14095-14107. https://doi.org/10.1103/PhysRevB.61.14095.

[40] C. Mao, M. Wood, L. David, S.J. An, Y. Sheng, Z. Du, H.M. Meyer, R.E. Ruther, D.L. Wood, Selecting the Best Graphite for Long-Life, High-Energy Li-Ion Batteries, J. Electrochem. Soc. 165 (2018) A1837-A1845. https://doi.org/10.1149/2.1111809jes.

[41] A. Sadezky, H. Muckenhuber, H. Grothe, R. Niessner, U. Pöschl, Raman microspectroscopy of soot and related carbonaceous materials: Spectral analysis and structural information, Carbon N. Y. 43 (2005) 1731-1742. https://doi.org/https://doi.org/10.1016/j.carbon.2005.02.018.

[42] X. Dou, I. Hasa, D. Saurel, C. Vaalma, L. Wu, D. Buchholz, D. Bresser, S. Komaba, S. Passerini, Hard carbons for sodium-ion batteries: Structure, analysis, sustainability, and electrochemistry, Mater. Today. 23 (2019) 87-104. https://doi.org/https://doi.org/10.1016/j.mattod.2018.12.040.

[43] M.A. Pimenta, G. Dresselhaus, M.S. Dresselhaus, L.G. Cançado, A. Jorio, R. Saito, Studying disorder in graphite-based systems by Raman spectroscopy, Phys. Chem. Chem. Phys. 9 (2007) 
1276-1291. https://doi.org/10.1039/b613962k.

[44] E.A. Olivetti, G. Ceder, G.G. Gaustad, X. Fu, Lithium-Ion Battery Supply Chain Considerations: Analysis of Potential Bottlenecks in Critical Metals, Joule. 1 (2017) 229-243.

https://doi.org/10.1016/j.joule.2017.08.019.

[45] Y.-K. Sun, D.-J. Lee, Y.J. Lee, Z. Chen, S.-T. Myung, Cobalt-Free Nickel Rich Layered Oxide Cathodes for Lithium-Ion Batteries, ACS Appl. Mater. Interfaces. 5 (2013) 11434-11440.

https://doi.org/10.1021/am403684z.

[46] Y. Kobayashi, M. Tabuchi, H. Miyashiro, N. Kuriyama, A new design of highly reversible LiNiO2: Defect formation in transition metal site, J. Power Sources. 364 (2017) 156-162. https://doi.org/10.1016/j.jpowsour.2017.08.027.

[47] W. Li, J.N. Reimers, J.R. Dahn, In situ x-ray diffraction and electrochemical studies of Li1-xNiO2, Solid State Ionics. 67 (1993) 123-130. https://doi.org/10.1016/0167-2738(93)90317-V.

[48] H. Li, N. Zhang, J. Li, J.R. Dahn, Updating the Structure and Electrochemistry of Li x NiO 2 for $0 \leq x$ $\leq 1$, J. Electrochem. Soc. 165 (2018) A2985-A2993. https://doi.org/10.1149/2.0381813jes.

[49] T. Ohzuku, A. Ueda, M. Nagayama, Electrochemistry and Structural Chemistry of LiNiO2 (R3m) for 4 Volt Secondary Lithium Cells, J. Electrochem. Soc. 140 (1993) 1862-1870. https://doi.org/10.1149/1.2220730.

[50] C. Delmas, J.P. Pérès, A. Rougier, A. Demourgues, F. Weill, A. Chadwick, M. Broussely, F. Perton, P. Biensan, P. Willmann, On the behavior of the LixNiO2 system: an electrochemical and structural overview, J. Power Sources. 68 (1997) 120-125. https://doi.org/10.1016/S03787753(97)02664-5.

[51] L. Croguennec, C. Pouillerie, A.N. Mansour, C. Delmas, Structural characterisation of the highly deintercalated LixNi1.02O2 phases (with x $\leq 0.30$ ), J. Mater. Chem. 11 (2001) 131-141. https://doi.org/10.1039/b003377o.

[52] L. De Biasi, A.O. Kondrakov, H. Geßwein, T. Brezesinski, P. Hartmann, J. Janek, Between Scylla and Charybdis: Balancing among Structural Stability and Energy Density of Layered NCM Cathode Materials for Advanced Lithium-Ion Batteries, J. Phys. Chem. C. 121 (2017) 26163-26171. https://doi.org/10.1021/acs.jpcc.7b06363.

[53] W.S. Yoon, K.Y. Chung, J. McBreen, X.Q. Yang, A comparative study on structural changes of LiCo1/3Ni1/3Mn1/3O2 and LiNi0.8Co0.15Al0.05O2 during first charge using in situ XRD, Electrochem. Commun. 8 (2006) 1257-1262. https://doi.org/10.1016/j.elecom.2006.06.005.

[54] C.S. Yoon, D.W. Jun, S.T. Myung, Y.K. Sun, Structural Stability of LiNiO2 Cycled above 4.2 v, ACS Energy Lett. 2 (2017) 1150-1155. https://doi.org/10.1021/acsenergylett.7b00304.

[55] V.I. Korepanov, Asymmetric least-squares baseline algorithm with peak screening for automatic processing of the Raman spectra, J. Raman Spectrosc. 51 (2020) 2061-2065. https://doi.org/10.1002/jrs.5952.

[56] M.N. Schmidt, T.S. Alstrøm, PEAK DETECTION AND BASELINE CORRECTION USING A CONVOLUTIONAL NEURAL NETWORK, (2019) 2757-2761.

https://doi.org/10.1109/ICASSP.2019.8682311. 
[57] J. Felten, H. Hall, J. Jaumot, R. Tauler, A. De Juan, A. Gorzsás, Vibrational spectroscopic image analysis of biological material using multivariate curve resolution-alternating least squares (MCRALS), Nat. Protoc. 10 (2015) 217-240. https://doi.org/10.1038/nprot.2015.008.

[58] V.P. Pauca, J. Piper, R.J. Plemmons, Nonnegative matrix factorization for spectral data analysis, Linear Algebra Appl. 416 (2006) 29-47. https://doi.org/10.1016/j.laa.2005.06.025.

[59] C.A. Meza Ramirez, M. Greenop, L. Ashton, I. ur Rehman, Applications of machine learning in spectroscopy, Appl. Spectrosc. Rev. 0 (2020) 1-31.

https://doi.org/10.1080/05704928.2020.1859525. 
